\title{
Sepsis - thoughtful management for the non-expert
}

\author{
Authors: Robert Tidswell ${ }^{A}$ and Mervyn Singer ${ }^{B}$
}

Sepsis is a common and often devastating medical emergency with a high mortality rate and, in many survivors, long-term morbidity. It is defined as the dysregulated host response to infection resulting in organ dysfunction, and its incidence is increasing as the population ages. However, it is a treatable and potentially reversible condition, especially if identified and treated promptly. A sound understanding of sepsis is crucial for optimal care. Although general guidelines are available for management, here we provide a foundation of understanding to encourage thoughtful, personalised management of sepsis during the acute phase. We provide an overview of the epidemiology, the new Sepsis-3 definitions, pathophysiology, clinical presentations, and investigation and management of sepsis for the non-expert.

\section{Introduction}

Hippocrates described sepsis in his Corpus Hippocratum, circa 400 BC, as a biological decay that leads to 'auto-intoxication', a definition which has changed little today. ${ }^{1}$ However, only over the past century have we begun to elucidate the nuances of a highly conserved and complex process.

Sepsis is a common and potentially catastrophic medical emergency. Reflecting Hippocrates, the new Sepsis-3 consensus statement defines it as 'life-threatening organ dysfunction caused by a dysregulated host response to infection'. 2 This new definition reflects the importance of host factors as well as the infecting pathogen and de-emphasises the prior focus on the systemic inflammatory response syndrome. To die from infection requires the development of organ failure (ie sepsis).

All doctors will be confronted by patients with sepsis. Robust data regarding sepsis epidemiology, both incidence and mortality, are elusive and are confounded by inconsistent coding. Patients with terminal conditions, such as cancer and end-stage respiratory disease, are susceptible to infection and often succumb to sepsis; these deaths relate more to the underlying disease process, rather than the sepsis per se. Given that most patients who are actively treated for sepsis will be admitted to intensive care, this probably

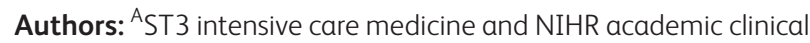
fellow, Bloomsbury Institute of Intensive Care Medicine, University College London, London, UK; ${ }^{B}$ professor of intensive care medicine, Bloomsbury Institute of Intensive Care Medicine, University College London, London, UK reflects a better guide to actual numbers. In 2012, there were 36,000 admissions with likely sepsis to English intensive care units (ICUs), of whom $32 \%$ died in hospital. ${ }^{3}$ From US hospital population databases, $4-5 \%$ of patients cultured and treated for infection died in hospital, although whether sepsis was the cause of death is unknown. ${ }^{4}$ Although mortality rates from sepsis are improving, the large increase in the denominator (sepsis incidence) provides a distorted view of this improvement. ${ }^{3}$

\section{Sepsis definitions}

The original sepsis definition, developed in 1991 and largely unchanged in a 2001 iteration, described sepsis as infection in the presence of a systemic inflammatory response syndrome (SIRS); that is, two or more abnormal values of heart rate, blood pressure, temperature or white cell count. Severe sepsis described sepsis plus organ dysfunction, whereas septic shock was 'sepsis-induced hypotension persisting despite adequate fluid resuscitation' 5,6

\section{Key points}

Sepsis is the dysregulated host response to infection resulting in organ dysfunction

Sepsis is a common medical emergency with a high mortality rate - involve experienced clinicians and the intensive care team early on

Guidelines are only a guide - individual patient factors must be considered to avoid harm and optimise management

Assess, reassess and reassess again - sepsis is an evolving situation and requires close monitoring of the response to therapy

If unresponsive to initial antimicrobials, consider fungal, atypical, resistant or viral pathogens or sepsis mimics

Sepsis can present in myriad ways - always have sepsis in your differential for patients with new-onset organ dysfunction

KEYWORDS: sepsis, infection, acute medicine, intensive care medicine, septic shock, medical education 
Table 1. Sepsis-3 definitions of sepsis and septic shock $^{a}$

Sepsis Sepsis is defined as life-threatening organ dysfunction caused by a dysregulated host response to infection

Organ dysfunction is operationalised by an acute increase in SOFA score of $\geq 2$ from the patient's baseline level

Septic A subset of sepsis in which underlying circulatory and shock cellular and/or metabolic abnormalities are profound enough to substantially increase mortality

Shock is operationalised as persisting hypotension requiring vasopressors to maintain a mean arterial pressure $\geq 65 \mathrm{mmHg}$ plus a lactate $>2 \mathrm{mmol} / \mathrm{L}$ despite adequate fluid resuscitation

${ }^{a}$ Based on Singer et al, $2016^{2}$

Limitations with these definitions became increasingly obvious: for example, SIRS is not specific for infection and does not differentiate between a normal and pathological host response to infection. What constitutes 'organ dysfunction' and 'shock' was not precisely clarified and, therefore, variable interpretations resulted in wide reported incidences and mortality rates. ${ }^{3}$

The Third international consensus definitions on sepsis and septic shock (2016) describes sepsis as a dysregulated host response leading to life-threatening organ dysfunction (Table 1). ${ }^{2}$ The Sequential (or Sepsis-related) Organ Failure Assessment (SOFA) score (Table 2 ) is used to operationalise organ dysfunction, allowing better characterisation for research, coding and epidemiology. ${ }^{7}$ A change in SOFA score of $\geq 2$ reflects a mortality risk of $>10 \%$ in a general hospital population. In patients meeting the criteria for septic shock, mortality is $>40 \%$. Septic shock describes a subset of patients with sepsis with a worse prognosis. It represents a combination of circulatory (ie hypotension) and cellular and/or metabolic abnormalities (ie lactataemia) persisting despite adequate fluid resuscitation. A raised lactate does not simply denote increased anaerobic metabolism, but is a marker of cellular stress.
Table 3. Quick (q)SOFA score ${ }^{a}$

Respiratory rate $\geq 22$ breaths/min

Altered mentation $(\mathrm{GCS}<15)$

Systolic blood pressure $\leq 100 \mathrm{mmHg}$

Based on Singer et al, 2016. ${ }^{2}$

GCS = Glasgow Coma Score

The quick (q)SOFA score is not part of the new definition but was developed to enable a rapid ( $1 \mathrm{~min})$ assessment of an infected patient likely to have sepsis if they fulfil two or more of the three bedside criteria (Table 3). In the UK, National Early Warning Score (NEWS) is already used in most hospitals to identify at-risk patients; of note, the qSOFA components represent three of the seven NEWS criteria.

\section{Pathophysiology}

It is important to emphasise the distinction between infection and sepsis. Sepsis represents the dysregulated host response to infection, and not the infection itself. By contrast, infection can and does exist without sepsis.

The pathophysiology of sepsis is complex and incompletely understood. The initial infectious insult is recognised by pattern recognition receptors (PRRs) within the innate immune system. These can be pathogen-related markers, termed 'pathogenassociated molecular patterns' (PAMPs), such as endotoxin, a lipopolysaccharide constituent of the cell wall of all Gramnegative bacteria. PRRs also recognise host cell contents, such as mitochondria and DNA, released in response to damage to the cells. These are termed 'damage-associated molecular patterns' (DAMPs). Activation of PRRs by PAMPs and DAMPs triggers the increased transcription and production of a range of pro- and antiinflammatory mediators, including multiple cytokines (eg tumor necrosis alpha (TNF- $\alpha$ ) and interleukins, such as IL-1, IL- 6 and IL-10), nitric oxide and eicosanoids from activated immune cells, endothelium and other cell types. ${ }^{8}$

Proinflammatory mediators initially predominate but, in most cases, are superseded by the anti-inflammatory response. This

Table 2. Sequential (or Sepsis-related) Organ Failure Assessment (SOFA) Score ${ }^{a}$

\begin{tabular}{|c|c|c|c|c|c|c|}
\hline \multirow[t]{2}{*}{ System } & \multicolumn{6}{|c|}{ Score } \\
\hline & & 0 & 1 & 2 & 3 & 4 \\
\hline Respiratory & $\mathrm{PaO}_{2} / \mathrm{FiO}_{2}(\mathrm{kPa})$ & $>53.3$ & $<53.3$ & $<40$ & $\begin{array}{l}<26.7 \text { plus respiratory } \\
\text { support }\end{array}$ & $\begin{array}{l}<13.3 \text { plus respiratory } \\
\text { support }\end{array}$ \\
\hline Coagulation & Platelets $\left(\times 10^{3} / \mu \mathrm{L}\right)$ & $\geq 150$ & $<150$ & $<100$ & $<50$ & $<20$ \\
\hline Liver & Bilirubin $(\mu \mathrm{mol} / \mathrm{L})$ & $<20$ & $20-32$ & 33-101 & $102-204$ & $>204$ \\
\hline Cardiovascular & $\begin{array}{l}\operatorname{MAP}(\mathrm{mmHg})(\mu \mathrm{g} / \mathrm{kg} / \\
\min )\end{array}$ & $>70$ & $\geq 70$ & $\begin{array}{l}\text { Dopamine }<5 \text { or } \\
\text { dobutamine at } \\
\text { any dose }\end{array}$ & $\begin{array}{l}\text { Dopamine } 5.1-15 \text { or } \\
\text { epinephrine } \leq 0.1 \text { or } \\
\text { norepinephrine } \leq 0.1\end{array}$ & $\begin{array}{l}\text { dopamine }>15 \text { or } \\
\text { epinephrine }>0.1 \text { or } \\
\text { norepinephrine }>0.1\end{array}$ \\
\hline Neurological & GCS & 15 & $13-14$ & $10-12$ & $6-9$ & $<6$ \\
\hline \multirow[t]{2}{*}{ Renal } & Creatinine $(\mu \mathrm{mol} / \mathrm{L})$ & $<110$ & $110-170$ & $171-299$ & $300-440$ & $>440$ \\
\hline & \multicolumn{4}{|l|}{ Urine output (mL/day) } & $<500$ & $<200$ \\
\hline
\end{tabular}

${ }^{a}$ Adapted from Vincent et al, 1996. ${ }^{7}$

$\mathrm{FiO2}$ = fraction of inspired oxygen; $\mathrm{GCS}=$ Glasgow Coma Scale; $\mathrm{MAP}=$ mean arterial pressure; $\mathrm{PaO}_{2}=$ partial pressure of oxygen in arterial blood 
might even have happened by the time the patient is admitted to ICU. Although needed to dampen the fires of inflammation, the downregulation of the inflammatory and/or immune response often results in a state of 'immune paresis' with decreased antigen presentation by innate immune cells, and white cell (neutrophil, monocyte and lymphocyte) deactivation. This increases the susceptibility of the patient to secondary infection.

Rather than specifically targeting the infection, widespread activation of neutrophils and the endothelium can lead to the accumulation of white cells within body organs, with the release of proteases and oxidants that can damage the host cells. The passage of white cells from the circulation into the organs is assisted by increased 'capillary leak'. This also allows the increased movement of fluid into the extravascular compartments, resulting in oedema.

The exaggerated inflammatory immune response triggers either up- or downregulation of multiple pathways within the body, including cardiovascular, hormonal, coagulation, neural, metabolic and bioenergetic systems. The net consequence is organ dysfunction. Although the precise mechanisms underlying organ dysfunction are still to be ascertained, cell death is a rare and patchy phenomenon, so tissue hypoxia alone cannot explain organ failure. Mitochondrial dysfunction is increasingly implicated.

Various reasons have been identified, such as tissue hypoxia, downregulation of gene transcripts encoding mitochondrial proteins, inhibition of the electron transport chain by nitric oxide and related species, oxidant damage, and decreased activity because of hormonal changes (eg low levels of thyroid hormone).

Mitochondria have multiple critical roles within the cell, including the bulk of ATP production, intracellular calcium regulation and generation of reactive oxygen species. Given that cell death is unusual in organs affected by sepsis, a decrease in ATP production must be matched by a corresponding decrease in cellular metabolism. This metabolic shutdown, manifested as organ dysfunction, is akin to hibernation. This enables potential reversibility and suggests the possibility of a protective process.

\section{Clinical picture - source of infection}

Sepsis can result from any infection. The most common foci are respiratory tract $(64 \%)$, abdomen (20\%), bloodstream (15\%) and genitourinary tract (14\%). Of positive cultures in the UK, approximately two-thirds are Gram-negative and one-third Gram-positive bacteria. ${ }^{9}$ The most common pathogens are Staphylococcus aureus, Escherichia coli and Pseudomonas species. Fungal infection (usually Candida) as a trigger of sepsis is relatively rare in the UK, but should be considered in immunosuppressed patients and those with long periods of hospitalisation, especially those previously treated with multiple courses of antibiotic. ${ }^{10}$

Pseudomonal, enterococcal or Acinetobacter sepsis, abdominal sources of infection, acute kidney injury, cancer, heart failure, immunosuppression and cirrhosis are all independent predictors of poor outcome. ${ }^{9}$

\section{Clinical presentation and diagnosis}

The classic presentation of infection (ie pyrexia, tachycardia, a flushed appearance, malaise, and symptoms and signs pointing to the site of infection, such as purulent sputum and cough, pyuria or meningism) is often absent. This is especially true during the early stages of infection when the patient might manifest non-specific features, or in young or older patients who are not infrequently afebrile or even display hypothermic 'cold' sepsis. The patient might be acutely or chronically confused or obtunded and not able to provide a clear history. They might be agitated and uncooperative with examination and investigations. The source of infection might not be apparent, and should trigger further detailed investigations, such as cross-sectional imaging.

For the diagnosis of sepsis, the patient with suspected infection must also have evidence of new organ dysfunction (ie a change in SOFA score $\geq 2$ ). This will be suggested rapidly at the bedside by a qSOFA score $\geq 2$, or a NEWS score $\geq 5$, but even if the patient does not hit these targets and there is clinical concern, this should trigger appropriate blood tests and treatment. The presentation of organ dysfunction can also be variable. In some cases, cardiovascular features might dominate (eg hypotension), whereas, in others, there might be predominant respiratory dysfunction that manifests as tachypnoea and cyanosis. The patient might be oliguric related to a direct renal pathology and/ or impaired circulation, or have altered mental status because of one or more of an impaired circulation, hypoxaemia, and/ or hypercapnoea from respiratory failure, and a direct septic encephalopathy. See Fig 1 for system specific effects of sepsis.

Having the experience to diagnose sepsis on an initial assessment before formal investigations return is a crucial skill that comes with experience. Even the experienced clinician might miss the presence of infection and/or organ dysfunction.

\section{Host factors affecting sepsis}

Patients over the age of 65 have a relative risk of sepsis of 13 and a relative mortality risk of 2.3 compared with younger patients. Only $47 \%$ of older patients who survive their episode of sepsis will be alive at 2 years. ${ }^{11}$

Other risk factors for sepsis include immunocompromise, cancer, alcohol excess, non-white ethnicity, a family history of death from sepsis, and vitamin D deficiency. Premenopausal women and those who undergo appropriate vaccination show lower rates of death. $^{12}$

\section{Investigation}

Initial investigations includes:

$>$ a careful history, including contacts, travel and recent surgical or other procedures

> observations - a minimum of every 5 min initially, and before and after every intervention

> blood tests and arterial blood gas analysis to assess respiratory, renal and hepatic organ function, haematology and coagulation variables, acid/base disturbance and lactate

> chest X-ray plus further imaging (eg CT scan or ultrasonography) guided by signs and symptoms

> septic screen - culture of blood, ideally before antibiotics. Urgent microscopy and culture of any other potentially infected fluid (eg urine, pleural fluid, cerebrospinal fluid, ascites, diarrhoea, aspirated or drained pus from an abscess, or debrided tissue). 'Pan-culture' is not advised except in occult sepsis.

Diagnostic sleuthery might be required when the source is elusive or the history not forthcoming. Examples include necrotising pharyngitis (Vincent's angina); thrombophlebitis of the internal 
jugular vein (Lemierre's syndrome); subphrenic and hepatic abscesses; necrotising fasciitis; toxic shock syndrome from a retained tampon, a small cut or a surgical wound (classically nonpus forming).

Further investigations to consider include:

$>$ HIV serology

$>$ echocardiography

$>$ molecular diagnostics if specific infection suspected (eg urinary antigen for Legionella and Pneumococcus, viral PCR, galactomannan, $\beta$-D-glucan)

$>$ extended fungal and mycobacterial cultures

$>$ malaria and other tropical diseases

$>$ cross-sectional imaging.

Case series report that up to $40 \%$ of patients with an initial diagnosis of sepsis might have only 'possible sepsis', or an alternative diagnosis altogether. ${ }^{13-15}$ Initial sepsis treatment should be started as soon sepsis is suspected, but other possible diagnoses should be kept in mind; if no source is found or the patient is unresponsive to treatment or behaving atypically, septic mimics should be considered. These conditions can present in a similar fashion to sepsis but lack an infectious trigger. Examples include pancreatitis, systemic lupus erythematosus, anaphylaxis, haematological malignancy, drug overdose and reactions, macrophage activation syndromes and beriberi.

\section{Treatment}

Sepsis and septic shock are emergencies and should be treated promptly by experienced clinicians. Junior members of the team should seek senior help and the intensive care team should be enlisted early. The 2017 Surviving Sepsis Campaign (SSC) guidelines offer a comprehensive guide to the management of sepsis and septic shock (www.survivingsepsis.org). ${ }^{16}$ However, even the strong recommendations therein are mainly based on moderate to weak evidence. Importantly, the guidelines must only be used as a guideline and not as 'rules of stone'. Good sepsis management comes from patient-specific management and repeated reassessment of the evolving situation.

\section{Fluid therapy}

If there is evidence of circulatory collapse or tissue hypoperfusion, repeated boluses of crystalloid should be administered until hypovolaemia is corrected. There is no clear evidence regarding the superiority and/or inferiority of $0.9 \%$ saline versus lactatebuffered solutions, such as Hartmann's. However, one should be aware of the high chloride content of $0.9 \%$ saline and the risk of hyperchloraemic acidosis, and the possibility of increasing lactataemia with the use of lactate-containing solutions.

Starch-based colloids are now actively discouraged. Human albumin solution can be considered in patients requiring large volumes of fluid and blood transfusion given to correct anaemia (maintain haemoglobin $>70 \mathrm{~g} / \mathrm{L}$ ). A higher haemoglobin level can be targeted if the patient has a history of cardiorespiratory insufficiency.

Fluid should be administered in a process involving repeated assessment of response to therapy. Multiple fluid challenges, sometimes totalling several litres, might be necessary within a few hours. However, a proportion of patients might be nonresponsive to fluid either because of severe myocardial depression and/or excessive loss of vascular tone. No one variable can guide fluid administration but both under- and overfilling should be avoided. Static central venous pressure measurement is no longer recommended. A dynamic change in central venous pressure or, preferably, stroke volume (a Frank-Starling response) in response to a fluid bolus is a more reliable marker of the adequacy of fluid resuscitation. Performing a passive leg raise (if patient condition permits) allows an assessment of fluid responsiveness without committing to fluid administration. However, this can induce pain or distress causing a rise in blood pressure unconnected to an increase in venous return. Other markers, such as blood pressure, heart rate, urine output, conscious level, central venous saturations, lactate or base deficit, should also be taken into consideration.

\section{Antimicrobials}

Antibiotics should be administered promptly to patients with sepsis. The antimicrobial spectrum should aim to cover the likely pathogen. The spectrum might need to be broad, covering both Gram-positive and Gram-negative pathogens, if uncertain. Viral and fungal cover should be considered, if suspected. Nuanced consideration should be paid to patient-specific factors, such as historic microbiology results with known resistance patterns, meticillin-resistant Staphylococcus aureus (MRSA) status, and comorbidities, which predispose to specific pathogens (eg Pseudomonas in bronchiectasis). If in doubt, advice should be sought from a microbiology and/or infectious disease specialist. Cover can be narrowed when sensitivities and sources are identified. The impact of combination therapy (two antibiotics active against the same organism) is uncertain.

Optimal antibiotic duration remains contentious and few prospective randomised controlled trials have been performed. The SSC guidelines suggest 7-10 days, although the underpinning evidence base is weak. Of note, a trial in patients with complex intra-abdominal infection and (crucially) good source control showed that a median 4-day course resulted in excellent outcomes. ${ }^{17}$ Non-controlled data from Denmark and the UK showed a median 4-6-day course of antimicrobial therapy was associated with good outcomes and low rates of relapse. ${ }^{18,19}$

\section{Source control}

Adequate and early source control is key to improving outcomes. ${ }^{20}$ Abscesses should be drained and any perforated viscus repaired or isolated.

\section{Vasoactive drugs}

Patients with circulatory failure despite fluid therapy require vasoactive drug support. This is usually administered in an ICU setting, but can be started beforehand while transfer is being arranged. Norepinephrine is the recommended first-line vasopressor for patients with hypotension and a high cardiac output who have lost vascular tone. Vasopressin is a second-line treatment. A blood pressure should be targeted that is sufficient to achieve a presumed adequate perfusion pressure. This often depends on the patient's premorbid blood pressure. Current SSC guidelines recommend mean arterial pressure (MAP) values of at least $65 \mathrm{mmHg}$; however, the detrimental effects of excessive catecholamines must be weighed against potential organ 


Endocrine system
Cortisol - initial elevated cortisol release with
subsequent suppression
Thyroid - deranged TSH release and/or lower
thyroid hormone levels
Insulin - hyperglycaemia from gluconeogenesis,
glycogenolysis and relative insulin resistance
Pineal - loss of normal circadian melatonin releas

\section{Cardiovascular system}

Cardiac - tachycardia and increased cardiac output to compensate for reduced afterload. Can develop septic cardiomyopathy

Peripheral - endothelial dysfunction (leaky capillaries), peripheral vasodilation and loss of tone with decreased afterload leading to circulatory collapse

Renal

Acute kidney injury caused by circulatory, inflammatory and mitochondrial abnormalities leading to microvascular and tubular dysfunction

Metabolism

Net catabolic state with muscle breakdown

Gluconeogenesis and glycogenolysis

Mitochondrial dysfunction

Musculoskeletal system

Severe myopathy if prolonged illness

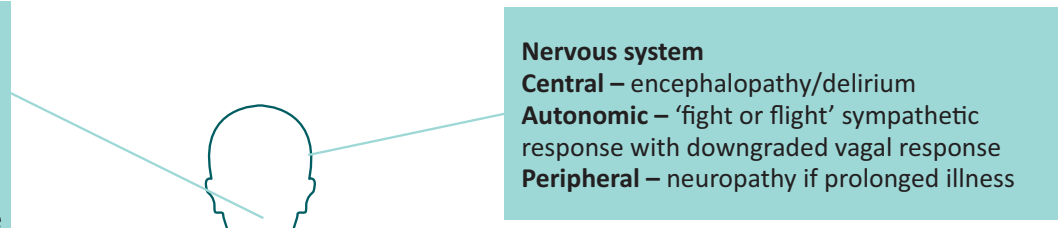

Respiratory system

Non-cardiogenic pulmonary oedema/acute

respiratory distress syndrome

Gastrointestinal system and liver Increased gut permeability and bacterial translocation

Stress ulceration of upper tract

Hepatic synthetic dysfunction and cholestasis

\section{Immune system}

An initial overall proinflammatory balance

followed by an overall anti-inflammatory state

with ensuing innate and adaptive immune

dysfunction and immune-tolerant state

\section{Haematological system}

Microthrombosis, fibrin deposition and, if

severe, disseminated intravascular coagulation hypoperfusion. Some patients with longstanding hypertension might require values above $70-75 \mathrm{mmHg}$, whereas other patients might adequately tolerate MAP values of $60 \mathrm{mmHg}$ or even lower.

However, vasopressors can compromise patients with sepsis and a low cardiac output related to a sepsis-induced myocardial depression. Alternatives that offer more inotropic activity include epinephrine, dobutamine and levosimendan, although these drugs can cause unwanted vasodilation and falls in blood pressure.

\section{Glucose control}

Glucose should be measured 1-2 hourly and insulin started to reach a target glucose level of $6.1-10 \mathrm{mmol} / \mathrm{L}$ after two consecutive readings above $10 \mathrm{mmol} / \mathrm{L}$. Care should be taken to avoid hypoglycaemia.

\section{Other organ support}

Respiratory support, either supplemental oxygen, non-invasive or invasive ventilation, is often required in the management of sepsis. Acute respiratory distress syndrome, manifesting as bilateral alveolar infiltrates resulting from non-cardiogenic pulmonary oedema and a deranged $\mathrm{PaO}_{2}: \mathrm{FiO}_{2}$ ratio, is often triggered by infection and represents the severe pulmonary manifestation of organ dysfunction. Mechanical ventilation with airway protection might also be needed if the patient is very obtunded or highly agitated. Renal replacement therapy will be needed to support the failing kidney when there is hyperkalaemia, fluid overload and/ or significant azotaemia or acidosis. Correction of coagulopathy should be guided by a haematologist, with appropriate coagulation factor concentrates and platelet levels maintained above $20 \times 10^{9} / \mathrm{L}$, in the absence of bleeding.

\section{Steroids and immunomodulatory therapies}

Hydrocortisone $50 \mathrm{mg}$ qds can be considered if there is ongoing haemodynamic instability despite high doses of vasopressors.

Some specific infections warrant the use of high-dose steroids, such as bacterial meningitis, Pneumocystis jirovecii pneumonia and miliary tuberculosis. ${ }^{21}$ Many other anti-inflammatory strategies have been trialled, but none have proved successful, in large part because of poor targeting of appropriate patients and suboptimal drug dosing and/or duration. Given that patients with sepsis often become immune suppressed, consideration is now being given to immunostimulatory therapy in this specific subset.

\section{Sepsis and comorbidities}

Some comorbidities change the nature of sepsis and can make guidelines less appropriate and, in some cases, potentially harmful. Some considerations and alterations to management are outlined below (Fig 2). 
Patient group

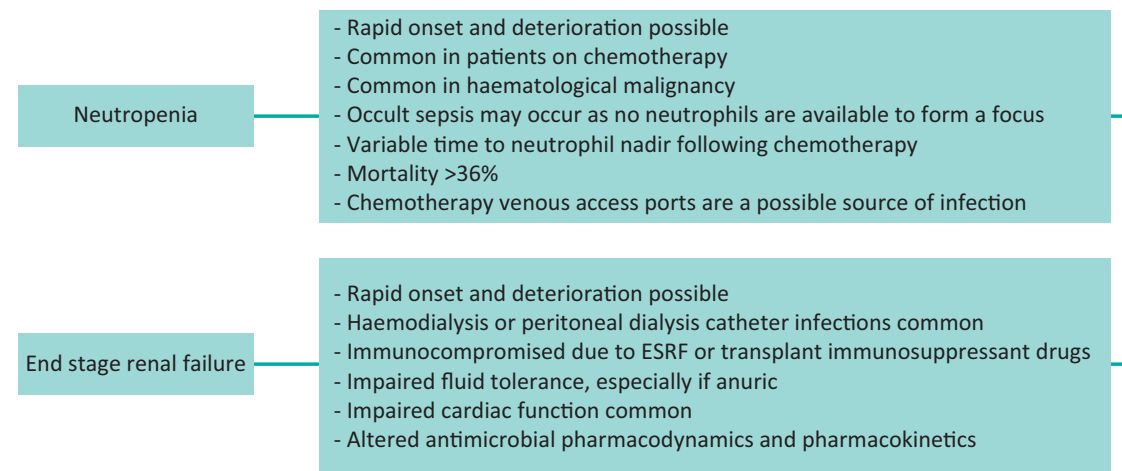

- Rapid onset and deterioration possible

- Multiple causes including: HIV, malignancy, autoimmune disease, primary

immunodeficiencies, transplant patients

- Consider occult and unusual presentations of sepsis

- Consider atypical, fungal and viral pathogens

\section{Potential alterations to management}

- Low threshold for suspicion of sepsis
- May benefit from G-CSF or white cell
transfusion
- Reverse barrier nursing required
- Paired cultures of vascular access lines and
removal if possible

Low threshold for suspicion of sepsis

Paired cultures of vascular access lines and removal if possible

Measure immunosuppressant drug levels

- Cautious fluid therapy

Cover nosocomial pathogens if attending

in-hospital dialysis

Low threshold for suspicion of sepsis - Consider performing an atypical screen, viral $\mathrm{PCR}$, galactomannan and $\beta$-D-glucan Escalate to ICU team early

Immunosuppressed

- Neutrophilia on full blood count may be normal

- Increased risk of hyperglycaemia

Abrupt cessation of steroids may precipitate hypoadrenalism

- Consider occult and masked presentations of sepsis

Rapid onset and deterioration possible

Highly susceptible to organ failure - encephalopathy, AKI, coagulopathy

Occult sepsis from bacterial translocation from the gut may occur

- May precipitate variceal haemorrhage

- Immunocompromised by reticuloendothelial dysfunction

- Consider spontaneous bacterial peritonitis (SBP)

Comorbidities common, especially cardiomyopathy

- Rapid onset and deterioration possible

- Common in sickle cell disease

- Impaired humoral immunity

- Susceptible to infection from encapsulated bacteria
- Low threshold for suspicion of sepsis

- Give IV hydrocortisone

- Monitor glucose levels

Fig 2. Considerations for sepsis management in specific patient groups. AKI = acute kidney injury; ESRF = end stage renal failure; ICU = intensive care unit; IV = intravenous

\section{Goals of care}

As the population lives longer with increasingly complex comorbidities and expectations, establishing realistic goals with patients and families is crucial. Decisions regarding appropriate escalation of care should be taken early and revisited regularly by senior clinicians and discussed with the family.

\section{The future of sepsis management}

Mortality and morbidity from sepsis remains high, and no effective novel therapies have been identified in any of the large randomised clinical trials since the discovery of antibiotics.

Current areas of interest include cytokine removal from the plasma, beta-adrenergic blockade, antibiotic infusions versus intermittent administration, vitamin D supplementation, and various immunostimulatory therapies, such as anti-PD1, GM-
CSF, and IL-7. Rehabilitation strategies to accelerate recovery are also being investigated. Further improvements in outcomes are likely to come from personalised medicine, including better diagnostics.

The dysregulated host response could be identified before the patient becomes critically ill. Rapid molecular diagnostics for pathogen identification will target more appropriate antibiotic therapy. The patient's biological phenotype can be accurately characterised and appropriately treated, ideally with rapid, point-of-care testing, to offer rapid identification and optimal dose titration. This approach could identify, for example, patients who will respond positively to corticosteroids, beta-blockers or immunostimulants.

The advent of 'Big data' is also beginning to usher in a new and interesting understanding of sepsis at a population level, which could yield important insights. 


\section{References}

1 Parillo JE, Kumar A. Historical aspects of critical illness and critical care medicine. Philadelphia: Saunders, 2009.

2 Singer M, Deutschman CS, Seymour CW et al. The Third International Consensus Definitions for Sepsis and Septic Shock (Sepsis-3). JAMA 2016:315:801-10.

3 Shankar-Hari M, Phillips GS, Levy ML et al. Developing a new definition and assessing new clinical criteria for septic shock. JAMA 2016;315:775

4 Seymour CW, Liu VX, Iwashyna T] et al. Assessment of clinical criteria for sepsis. JAMA 2016;315:762.

5 Bone RC, Balk RA, Cerra FB et al. Definitions for sepsis and organ failure and guidelines for the use of innovative therapies in sepsis. Chest 1992:101:1644-55.

6 Levy MM, Fink MP, Marshall JC et al. 2001 SCCM/ESICM/ACCP/ ATS/SIS International Sepsis Definitions Conference. Intensive Care Med 2003:29:530-8.

7 Vincent J-L, Moreno R, Takala J et al. The SOFA (Sepsis-related Organ Failure Assessment) score to describe organ dysfunction/ failure. Intensive Care Med 1996:22:707-10.

8 Takeuchi O, Akira S, Li S et al. Pattern recognition receptors and inflammation. Cell 2010:140(6):805-20.

9 Vincent J, Rello J, Marshall ] et al. International study of the prevalence and outcomes of infection in intensive care units. JAMA 2009;302(21):2323-9.

10 Harrison D, Muskett $\mathrm{H}$, Harvey S et al. Development and validation of a risk model for identification of non-neutropenic, critically ill adult patients at high risk of invasive Candida infection: the Fungal Infection Risk Evaluation (FIRE) Study. Health Technol Assess 2013;17:1-156.

11 Lemay AC, Anzueto A, Restrepo MI, Mortensen EM. Predictors of long-term mortality after severe sepsis in the elderly. Am J Med Sci 2014:347:282-8

12 Gotts JE, Matthay MA. Sepsis: pathophysiology and clinical management. BMJ. 2016;353:11585.
13 Long B, Koyfman A. Clinical mimics: an emergency medicinefocused review of sepsis mimics. J Emerg Med 2017;52:34-42.

14 Contou D, Roux D, Jochmans S et al. Septic shock with no diagnosis at 24 hours: a pragmatic multicenter prospective cohort study. Crit Care 2016;20:360.

15 Klein Klouwenberg PMC, Cremer OL, van Vught LA et al. Likelihood of infection in patients with presumed sepsis at the time of intensive care unit admission: a cohort study. Crit Care 2015;19:319.

16 Rhodes A, Evans LE, Alhazzani W et al. Surviving Sepsis Campaign: international guidelines for management of sepsis and septic shock: 2016. Intensive Care Med 2017:43:304-77.

17 Guidry CA, Sawyer RG. Short-course antimicrobial therapy for intraabdominal infection. N Engl J Med 2015;373:1577-8.

18 Jensen JU, Hein L, Lundgren B et al. Procalcitonin-guided interventions against infections to increase early appropriate antibiotics and improve survival in the intensive care unit: a randomized trial. Crit Care Med 2011;39:2048-58.

19 De Santis V Gresoiu M, Corona A, Wilson APR, Singer M. Bacteraemia incidence, causative organisms and resistance patterns, antibiotic strategies and outcomes in a single university hospital ICU: continuing improvement between 2000 and 2013. J Antimicrob Chemother 2015;70:273-8.

20 Bloos F, Thomas-Rüddel D, Rüddel H et al. Impact of compliance with infection management guidelines on outcome in patients with severe sepsis: a prospective observational multi-center study. Crit Care 2014;18:R42.

21 Brouwer MC, McIntyre P, Prasad K, van de Beek D. Corticosteroids for acute bacterial meningitis. Cochrane Database Syst Rev 2015:CD004405.

Address for correspondence: Dr Robert Tidswell, University College London, Cruciform Building, Gower Street, London WC1E 6BT, UK.

Email: tidswell.robert@gmail.com 